\title{
Fluctuations in interbank network dynamics
}

\author{
Daniel O. Cajueiro, ${ }^{1}$ Benjamin M. Tabak, ${ }^{2}$ and Roberto F. S. Andrade ${ }^{3}$ \\ ${ }^{1}$ Departmento de Economia, Universidade de Brasília, 70910-900 Brasília, Brazil \\ ${ }^{2}$ Universidade de Catálica de Brasília, 70790-160 Brasília, Brazil \\ ${ }^{3}$ Instituto de Física, Universidade Federal da Bahia, 40210-340 Salvador, Brazil
}

(Received 18 July 2008; revised manuscript received 22 December 2008; published 18 March 2009)

\begin{abstract}
This work investigates the scaling properties of fluctuations in the flux of individual agents with respect to their average flux in an interbank network. The analyzed data provide information on daily values of $f_{i}^{\text {asset }}$, the credit provided by bank $i$ in the interbank network, and $f_{i}^{\text {liab }}$, the credit received by bank $i$ from the other banks of the network. The investigation focuses on the scaling properties of the fluctuations in the raw data $f_{i}^{\text {asset }}, f_{i}^{\text {liab }}$, and $f_{R, i}^{\text {ext }}(t)=f_{i}^{\text {asset }}-f_{i}^{\text {liab }}$, as well as on similar properties internal and external fluctuations $f_{i}^{\text {int }}$ and $f_{i}^{\text {ext }}$, which are derived according to a recently proposed methodology [M. Argollo de Menezes and A. L. Barabasi, Phys. Rev. Lett. 93, 068701 (2004)]. Finally, a "rolling sampling" approach is introduced in order to deal with the nonstationarity of the fluxes. The results suggest that exponents are time varying, hinting that the considered interbank network is changing with time.
\end{abstract}

DOI: 10.1103/PhysRevE.79.037101

PACS number(s): 89.65.-s, 89.75.Fb, 89.75.Hc

In recent years, one of the main issues discussed in the statistical physics literature is related to the dynamics of complex systems such as airports [1], financial institutions $[2,3]$, web pages [4], social networks [5-7], and chaotic interacting functions [8]. All of them share several common features, among which being associated to network structures is the most relevant one for the current work. Several comprehensive reviews on this subject are now available [9-11].

For the purpose of characterizing dynamical processes taking place on such structures, de Menezes and Barabasi [12] investigated the general problem of the coupling between the time average flux $\left\langle f_{i}\right\rangle$ and dispersion $\sigma_{i}$ $=\sqrt{\left\langle\left(f_{i}-\left\langle f_{i}\right\rangle\right)^{2}\right\rangle}$ of individual nodes. Their results indicate that, for five different systems, the dispersion of the flux depends on the average flux as a power law,

$$
\sigma_{i} \sim\left\langle f_{i}\right\rangle^{\alpha}
$$

The authors found that these systems can be classified into two different classes depending on the value of $\alpha$, namely, systems with $\alpha \approx \frac{1}{2}$ and systems with $\alpha \approx 1$. While the main characteristic of systems with $\alpha \approx \frac{1}{2}$ seemed to be the absence of relevant external dynamics, the opposite happens to systems with $\alpha \approx 1$. Since almost all data sets provide information on the time dependence of the total flux $f_{i}(t)$ on each node, the same authors proposed a definition to separate internal $f_{i}^{\text {int }}(t)$ from external $f_{i}^{\text {ext }}(t)$ contributions of the total flux [13], so that it is possible to write

$$
f_{i}(t)=f_{i}^{\mathrm{int}}(t)+f_{i}^{\mathrm{ext}}(t) .
$$

In spite of the fact that the results reported in the quoted works are consistent, it is necessary to check whether the same scaling behavior and flux separation is found in other similar systems.

In the present work, we consider a data set with information of daily fluxes of the interbank network provided by the Brazilian Central Bank. Contrary to the data sets considered in the quoted works $[12,13]$, we obtain direct information on two independent fluxes: $f_{i}^{\text {asset }}$, the credit provided by bank $i$ in the interbank network, and $f_{i}^{\text {liab }}$, the credit received by bank $i$ from the other banks of the network. The larger amount of information allows for the investigation of the scaling properties of the data, as well as to test whether it is compatible with the proposed decoupling into internal and external flux contributions. We define the raw data external flux by

$$
f_{i}^{\mathrm{asset}}(t)=f_{i}^{\mathrm{liab}}(t)+f_{R, i}^{\mathrm{ext}}(t)
$$

as a residual variable that balances the two known contributions. Let us recall that banks may fund their interbank activities with their own interbank liabilities. However, if interbank assets exceed interbank liabilities then external fluxes are positive, which suggests that there is an excess demand of interbank credit and banks have to channel funds from other sources. On the other hand, if interbank liabilities are larger than interbank assets, then the external flux is negative and banks are channeling funds to other sources. Equation (3) is the flux form of the fundamental equation from accounting theory: assets $_{t}=$ liabilities $_{t}+$ net worth $_{t}$, which implies that the source of funding (liabilities and net worth) of a specific firm is equal to its investments (assets).

Although a formal analogy between Eqs. (2) and (3) seems straightforward $\left(f_{i}^{\text {asset }} \Leftrightarrow f_{i}, f_{i}^{\text {liab }} \Leftrightarrow f_{i}^{\text {int }}, f_{R, i}^{\text {ext }} \Leftrightarrow f_{i}^{\text {ext }}\right)$, the scaling results obtained in this study show that the definitions for decoupling internal and external fluctuations are of different nature.

We show that there are scaling laws such as the one presented in Eq. (1) among the dispersions $\sigma^{\text {asset }}, \sigma^{\text {liab }}, \sigma_{R}^{\text {ext }}, \sigma^{\text {int }}$, and $\sigma^{\text {ext }}$ and the corresponding average flux $\left\langle f_{i}^{\text {asset }}\right\rangle$, with exponents given, respectively, by $\alpha^{\text {asset }}, \alpha^{\text {liab }}, \alpha_{R}^{\text {ext }}, \alpha^{\text {int }}$, and $\alpha^{\text {ext }}$. Furthermore, we go a step ahead presenting empirical evidence that these exponents are time varying, which implies structural changes in the fundamentals of the network.

Before we discuss the main results of our study, let us mention that related contributions to this work have been obtained for several distinct complex systems. In $[14,15]$, the 
multiscaling behavior in the dynamics of fluctuations is discussed. While Eisler et al. [14] found anomalous values of $\alpha$ (values different from $1 / 2$ and 1) for fluctuations of stock market activity, Eisler and Kertesz [15] proposed a formalism to capture anomalous values of $\alpha$ that are consequence of two different phenomena: (a) the coupling between a variable called impact and the degree of the visited node and (b) the dependence of the number of visitations on the degree of the node. Moreover, in [14], $\alpha$ is shown to be time scale dependent. Finally Duch and Arenas [16] presented evidence questioning the existence of universality classes in $N$ queue systems of type $M / M / 1$.

In the context of specific aspects of finance dynamics, this work also contributes to a better understanding of banking networks such as in [2,17-19]. In fact, the fluxes that will be studied here are extremely important since they are sources of risk in a banking system [20]. In particular, $f_{i}^{\text {asset }}$ measures the exposure of bank $i$ to the banking system; i.e., if a bank that borrowed money from bank $i$ bankrupts, the failure of that bank may also trigger the failure of bank $i$. This phenomenon, called financial contagion, has been considered for instance in [20-22].

The analysis performed in this work is based on information of all interbank exposures in the Brazilian interbank market. All financial institutions report their counterpart in the interbank market and their size exposure. Interbank assets represent approximately $10 \%-15 \%$ of total banking assets within the period under analysis. Therefore, it is an important market where banks are able to finance themselves, at least in the short run. The average daily turnover of this market is around 10 billion dollars and stocks are around 200 billion dollars. The interbank data have been collected from January 2, 2004 to December 31, 2005 for all banks and financial institutions that have exposures in the interbank market in the period of analysis. The sample comprises of 86 banks and 23 nonbank financial institutions [23].

The decoupling of $f_{i}(t)$ into $f_{i}^{\text {int }}(t)$ and $f_{i}^{\text {ext }}(t)$ for all network nodes proceeds according to the definitions [13],

$$
f_{i}^{\text {ext }}(t)=A_{i} \sum_{i=1}^{N} f_{i}(t),
$$

where

$$
A_{i}=\frac{\sum_{t=1}^{T} f_{i}(t)}{\sum_{t=1}^{T} \sum_{i=1}^{N} f_{i}(t)},
$$

and

$$
f_{i}^{\mathrm{int}}(t)=f_{i}(t)-A_{i} \sum_{i=1}^{N} f_{i}(t) .
$$

For the raw data, we have found "static exponents" $\alpha^{\text {asset }}=0.82, \alpha^{\text {liab }}=0.50$, and $\alpha_{R}^{\text {ext }}=0.62$. These results were determined by the slope of the orthogonal least-squares straight lines presented in the first three panels of Fig. 1. Based on these results, one may note that two of the total

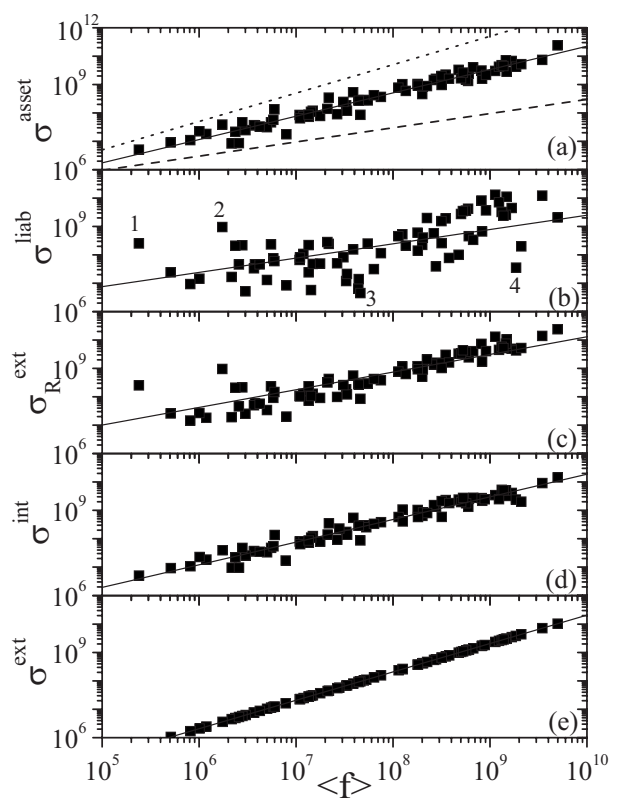

FIG. 1. Dependency of fluctuations on average flux. Panels (a)(e) show the dependence of $\sigma^{\text {asset }}, \sigma^{\text {liab }}, \sigma_{R}^{\text {ext }}, \sigma^{\text {int }}$, and $\sigma^{\text {ext }}$ as a function of $\left\langle f_{i}^{\text {asset }}\right\rangle$, respectively. Lines correspond to orthogonal least squares (OLS) values used to determine the dynamical exponents.

flux $\left\langle f_{i}\right\rangle$ present fractionary (anomalous) exponents that do not belong to the two universality classes $\alpha \approx 0.5$ and $\alpha$ $\approx 1.0$ introduced in [12]. Indeed, this behavior is much closer to those found for the fluxes in other financial network $[14,15]$. For the purpose of better displaying our results, we include in Fig. 1(a) straight lines with slopes corresponding to the two universality classes. In contrast, the results obtained for $\alpha^{\text {int }}=0.798$ and $\alpha^{\text {ext }}=1.0$ [Figs. 1(d) and 1(e)] differ substantially from those reported before for $\alpha^{\text {liab }}$ and $\alpha_{R}^{\text {ext }}$. In order to check the effect of the network heterogeneity, we also analyzed two subsamples that contain the 20 smallest and largest financial institutions. Due to the sparsity of links among the nodes of the first subsample, it does not provide any useful information. On the other hand, the second subsample reinforced our results for the full set, with similar fractional exponents.

These results indicate, at first sight, that the decoupling into internal and external contributions does not match the actual reported data for assets and liabilities. A closer analysis reveals that the points in Figs. 1(b) and 1(c) are much scattered as compared to those in Figs. 1(d) and 1(e). This includes, for instance, two points $[1$ and 2 in panel (b) $]$ in the region of smaller values of $\left\langle f_{i}\right\rangle$, for which $\sigma^{\text {liab }}, \sigma_{R}^{\text {ext }} \gg \sigma^{\text {asset }}$. The presence of such large contributions in both terms in right-hand side (rhs) of Eq. (3), but not in the total flux, indicates that the two contributions have opposite signs. Another example of actual behavior not recovered by Eqs. (2)-(6) refers to much smaller values of $\sigma^{\text {liab }}$ in comparison to $\sigma^{\text {asset }}$ [points 3 and 4 in panel (b)]. For such situations, the actually important contribution to $\sigma^{\text {asset }}$ stems from $\sigma_{R}^{\text {ext }}$. This suggests that Eqs. (2)-(6) are not able to capture such nonusual details of the actual data.

Small values of $\alpha^{\text {liab }}$ and $\alpha_{R}^{\text {ext }}$ are also due to the presence 


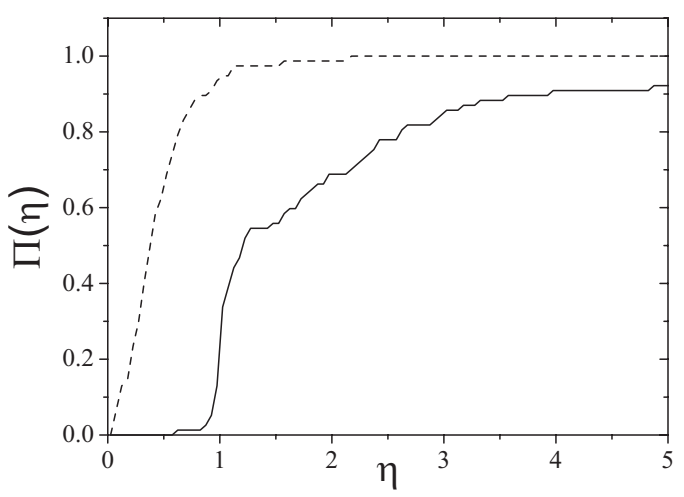

FIG. 2. Integrated probability density $\Pi\left(\eta_{i}\right)=\sum_{j=1}^{i} P\left(\eta_{j}\right)$ for $\eta$ $=\sigma_{R}^{\text {ext }} / \sigma^{\text {liab }}$ (solid line) and $\eta=\sigma^{\text {ext }} / \sigma^{\text {int }}$ (dashes). For the raw data analysis, external fluctuations dominate and $\alpha_{R}^{\text {ext }}$ is closer to $\alpha^{\text {asset }}$ than $\alpha^{\text {liab }}$. For the decoupling obtained by Eqs. (2)-(6), values of $\alpha^{\text {ext }}$ are overwhelmingly smaller than $\alpha^{l i a b}$, resulting in $\alpha_{\text {int }}$ $\approx \alpha^{\text {asset }}$

of outliers like points 1-4 indicated in Fig. 1(b). For instance, if points 1 and 2 are neglected in the evaluation of $\alpha$, these values change to $\alpha^{\text {liab }}=0.56$ and $\alpha_{R}^{\text {ext }}=0.67$. This is a further confirmation that $\alpha^{\text {liab }}$ and $\alpha_{R}^{\text {ext }}$ assume anomalous values as well. Despite these differences, let us notice that the value $\alpha^{\text {ext }}=1.0$ is in best accordance with the general feature observed for previous analysis of other data sets by the decoupling method.

The relation among the exponents describing the quantities in Eqs. (2) and (3) can be well explained by the relative magnitude of the three terms involved in each of these expressions. Indeed, as observed in [13], the probability distribution $P\left(\eta_{i}\right)$ of the variable $\eta_{i}=\sigma_{i}^{\text {ext }} / \sigma_{i}^{\text {liab }}$ may be used to determine whether the dynamics of $f^{\text {asset }}$ is mainly dominated by the dynamics of $f^{\text {liab }}$ or by the dynamics of $f^{\text {ext }}$. The graph of $P\left(\eta_{i}\right) \times \eta_{i}$ indicates that, in average, $f^{\text {ext }}$ is larger than the deviation of $f^{\text {liab }}$ with the distribution peaked around $\eta_{i}$ $\approx 1.2$. Thus, the dynamics of $f^{\text {ext }}$ has more impact on the fluctuations of $f$ than the dynamics of $f^{\text {liab }}$. This indicates that the channel of external sources of funding is very important in the Brazilian interbank network. On the other hand, the decoupling provided by Eq. (2) behaves all way around as $P\left(\eta_{i}\right)$ is peaked around the value $\eta_{i} \approx 0.3$. This explains why $\alpha^{\text {asset }}$ stays much closer to $\alpha^{\text {int }}$ than to $\alpha_{R}^{\text {ext }}$. Such properties are illustrated in Fig. 2, where we show the integrate probability density $P\left(\eta_{i}\right)=\sum_{j=1}^{i} P\left(\eta_{j}\right)$ for $\eta=\sigma_{R}^{\text {ext }} / \sigma^{\text {liab }}$ and $\eta$ $=\sigma^{\mathrm{ext}} / \sigma^{\mathrm{int}}$.

Let us now discuss the large scale time dependence of the fluxes in the data. As shown by Fig. 3, values of $f, f^{\text {int }}$, and $f^{\text {ext }}$ for a typical node present significant variations, suggesting that these flows are strongly nonstationary in the large scale regime. Moreover, we have observed that the time fluctuation pattern is node dependent. Therefore, one should consider the possibility that the previous result presented in Fig. 1 may have been affected by such nonstationary behavior. This topic was not considered in $[12,13]$ since the networks considered in these papers present quite stationary dynamics.

To deal with this issue, we have used a "rolling sampling" approach in a similar way as in previous papers by two of us $[24,25]$. In the study of world financial time series, it was

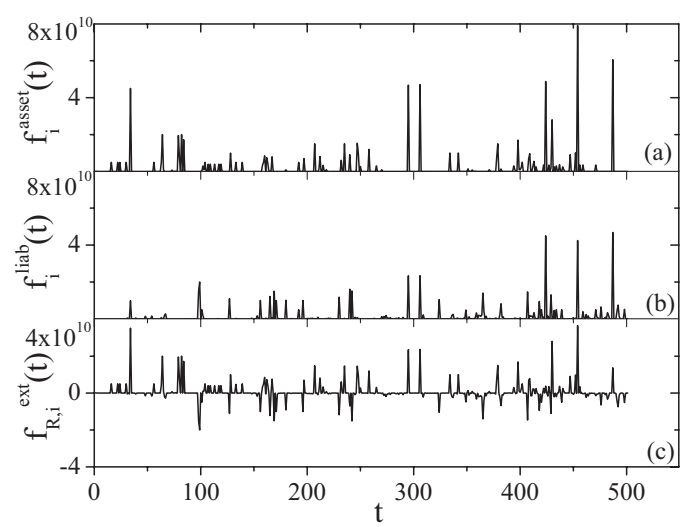

FIG. 3. The fluxes of the Brazilian interbank market over time for a typical node.

realized that a unique Hurst exponent $H$ was not enough to characterize the dynamics of financial markets since the system is time varying with a clear tendency to become more efficient. Based on that approach, time dependent values of $\alpha$ were evaluated within a window with a fixed length $L$, smaller than that of the total series. The process starts by evaluating $\alpha(t=L / 2)$ based on the first $L$ entries of the set. Then, we exclude the first entry and include $L+1$ th one, and the procedure is repeated to obtain $\alpha(t=L / 2+1)$. This process continues until all observations are exhausted. We employ sliding windows with $L=180$ days to perform our "rolling statistics." Figure 4 shows these results presenting the value of $\alpha$ over time for each flux. The results plotted in Fig. 4 show no evidence of the two standard universality classes in this system, what is corroborated by the time average values of the exponents, found to be $\overline{\alpha^{\text {asset }}}=0.864, \overline{\alpha^{\text {liab }}}=0.581$,

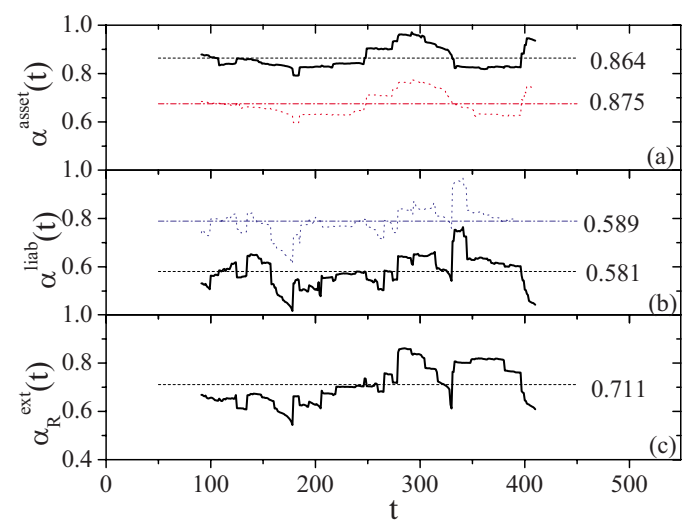

FIG. 4. (Color online) Time dependence of $\alpha(t)$ for the raw data fluxes of the Brazilian interbank network. Dashed lines correspond to indicated values of time average values of $\alpha(t)$. Histograms of values of $\alpha(t)$ (not shown) discard the possibility that the variation in the exponent over time is due to "measurement errors." This could be the case if the histograms had a normal distribution or at least a monomodal distribution. Dotted lines in (a) and (b) indicate local value of $\alpha(t)$ when the window width is changed to $L=160$ and 200, respectively. For the sake of better visualization, the points have been vertically displaced by -0.2 (a) and +0.2 (b). Standard deviations are 0.045 [(a), solid line], 0.047 [(a), dotted line], 0.064 [(b), solid line], 0.058 [(b), dotted line], and 0.078 [(c), solid line]. 
and $\overline{\alpha_{R}^{\mathrm{ext}}}=0.711$. Actually, the problem of choosing the optimal size of windows is not trivial. If small size windows are considered, the sample may not be enough to provide a good estimate of the scaling parameter. On the other hand, if the size of the windows is too large, the correct local dynamics of the parameter may not be perceived. The $L=180$ choice used in this work achieves a trade-off between these two factors. As also indicated in Fig. 4, the results are relatively robust if we compare them with those provided by windows with $10 \%$ width variation.

A very characteristic feature of the three series shown that is easily detected in the plots presented in Fig. 4 is the presence of structural breaks, a very clear mark that the fundamentals of the network are time varying. Such breaks can be traced back to sudden changes in the portfolio of only but a few institutions. Among them, changes are more drastic if smaller institutions borrow or lend money in a way that exceeds its solvency threshold. In such case, they contribute to change the disposition of the corresponding points in the $\sigma_{i}$ $\times f_{i}$ plots, much in the way as exemplified by points 1 and 2 in Fig. 1(a). Another source of sharp changes in the values of $\alpha$ is financial crisis of institutions. The bankruptcy of a medium size bank at $t=330$ may have caused changes in the network, and the peak in variables $\alpha^{\text {asset }}$ and $\alpha^{\text {ext }}$ coincides with this failure.
To conclude, in this work we have shown that the data of the fluxes in the Brazilian interbank network indicate that fluctuations in two of the three raw data components are not described by universal exponents proposed in [12]. The fluctuation decoupling approach leads to different exponents, but only one describing the external contributions matches the value proposed in [13]. Moreover, the two approaches assign different weights to the components into which fluctuations are decoupled. This clearly indicates that the assumptions which support the fluctuation decoupling framework must be carefully tested before the method is applied. In the considered analysis, the results indicated that the conditions are not met. Besides that, we investigated the time dependence of flux fluctuations for the raw data, finding time-varying exponents. Such results suggest that fundamentals of the network are changing over time.

The authors gratefully acknowledge research grants (Grants No. 306052/2007-5, No. 400655/2006-3, No. 308494/2006-7, and No. 304248/2007-0) from CNPQ (Brazilian agency). The first author is indebted to Professor M. Argollo De Menezes for helping him to understand some details of his paper [12].
[1] W. Li and X. Cai, Phys. Rev. E 69, 046106 (2004).

[2] G. Iori, G. De Masi, O. V. Precup, G. Gabbi, and G. Caldarelli, J. Econ. Dyn. Control 32, 259 (2008).

[3] G. Iori, R. Reno, G. De Masi, and G. Calderelli, Physica A 376, 467 (2007).

[4] R. Pastor-Satorras, A. Vazquez, and A. Vespignani, Phys. Rev. Lett. 87, 258701 (2001).

[5] A. Grabowski and R. A. Kosinski, Phys. Rev. E 73, 016135 (2006).

[6] D. O. Cajueiro, Phys. Rev. E 72, 047104 (2005).

[7] M. Boguña, R. Pastor-Satorras, A. Diaz-Guilera, and A. Arenas, Phys. Rev. E 70, 056122 (2004).

[8] E. P. Borges, D. O. Cajueiro, and R. F. S. Andrade, Eur. Phys. J. B 58, 469 (2007).

[9] R. Albert and A. L. Barabasi, Rev. Mod. Phys. 74, 47 (2002).

[10] S. Boccaletti, V. Latora, Y. Moreno, M. Chavez, and D. U. Hwang, Phys. Rep. 424, 175 (2006).

[11] L. D. Costa, F. A. Rodrigues, G. Travieso, and P. R. V. Boas, Adv. Phys. 56, 167 (2007).

[12] M. Argollo de Menezes and A. L. Barabasi, Phys. Rev. Lett. 92, 028701 (2004).
[13] M. Argollo de Menezes and A. L. Barabasi, Phys. Rev. Lett. 93, 068701 (2004).

[14] Z. Eisler, J. Kertesz, S. H. Yook, and A. L. Barabasi, Europhys. Lett. 69, 664 (2005).

[15] Z. Eisler and J. Kertesz, Phys. Rev. E 71, 057104 (2005).

[16] J. Duch and A. Arenas, Phys. Rev. Lett. 96, 218702 (2006).

[17] M. Boss, H. Elsinger, M. Summer, and S. Thurner, Quant. Finance 4, 677 (2004).

[18] Y. S. Wan, Z. Chen, and Z. R. Liu, Dyn. Cont. Disc. Imp. Syst.-Ser. B-Applic. and Alg. 13, 441 (2006).

[19] K. Soramaki, M. L. Bech, J. Arnold, R. J. Glass, and W. E. Beyeler, Physica A 379, 317 (2007).

[20] F. Allen and D. Gale, J. Polit. Econ. 108, 1 (2000).

[21] G. Iori and S. Jafarey, Physica A 299, 205 (2001).

[22] G. Iori, S. Jafarey, and F. G. Padilha, J. Econ. Behav. Organ. 61, 525 (2006).

[23] Confidentiality clauses in the Brazilian interbank market do not allow the disclosure of identification or further information on the financial agents included in the analyzed data set.

[24] D. O. Cajueiro and B. M. Tabak, Physica A 346, 577 (2005).

[25] D. O. Cajueiro and B. M. Tabak, Physica A 336, 521 (2004). 